\title{
Anodizing for Design and Function
}

\author{
Runge JM \\ Comprehensive Metallurgical Consulting, CompCote International
}

${ }^{*}$ Corresponding author: Runge JM, Ph.D., Comprehensive Metallurgical Consulting, CompCote International, Inc., Chicago, Illinois, USA, E-mail: jude.runge@compcote.com

Citation: Runge JM (2014) Anodizing for Design and Function. J Mater Sci Nanotechnol 1(1): S108. doi: 10.15744/2348-9812.1.S108

Received Date: June 03, 2014 Accepted Date: June 11, 2014 Published Date: August 01, 2014

\begin{abstract}
Two basic reactions occur during the anodizing of aluminum: 1) the aluminum is consumed and 2) an oxide grows. By accepting this statement as true, the anodizing process can be viewed as a corrosion process, and anodizing can be modeled using the Tafel Equation. Anodizing process parameters of electrolyte chemistry and concentration, temperature, aluminum substrate resistance and current density are presented as they relate to the Tafel Equation and how they impact the anodic aluminum oxide structure and properties. Understanding this relationship is consequent in making anodizing an engineering process, one that enables tuning the structure such that it yields distinct characteristics to fulfill design and application requirements.

Keywords: Anodizing; Corrosion; Anodic Aluminum Oxide; Aluminum Oxide; Oxide; Tafel Equation; Current Density; Oxidation Potential; Interfacial Resistance

Abbreviations: AAO-Anodic Aluminum Oxide
\end{abstract}

\section{Introduction}

Anodizing is a complex multidisciplinary process, encompassing chemical and electrochemical effects with physical metallurgy and engineering issues/procedures. The physical metallurgy effects, in particular, are not only electrochemical in nature but involve thermodynamics, which vary with alloy chemistry and the manufacturing process of the substrate. As by its very nature, anodizing involves metallic dissolution to produce an oxide, the various effects can be brought together within the realm of corrosion science.

A metal electrode is no longer at equilibrium (corrodes) when a net current flows [to or] from its surface [1]. Under most conditions, by controlling the current flow from the electrode, one can control the corrosion rate, and the development of the reaction product at the interface with the electrolyte. This is done during aluminum anodizing. Anodizing therefore, can be defined as a corrosion process.

The bridge between corrosion science and the anodizing process is the Tafel Equation.

$$
\mathrm{N}=\beta_{\mathrm{a}} \frac{\log \mathrm{i}_{\mathrm{a}}}{\mathrm{i}_{\mathrm{o}}}+\frac{2.3 \mathrm{RT}}{\mathrm{nF}} \log \frac{\left(1-\mathrm{i}_{\mathrm{a}}\right)}{\mathrm{i}_{\mathrm{o}}}
$$

Where $\mathrm{N}=$ oxidation polarization (volts)

$$
\begin{gathered}
\mathrm{i}_{\mathrm{a}}=\text { anodic current density (amps/unit area) } \\
\mathrm{i}_{\mathrm{o}}=\text { exchange current density }(\mathrm{amps} / \text { unit area) } \\
\beta_{\mathrm{a}}=\text { surface polarization slope (volts) } \\
\frac{2.3 \mathrm{RT}}{\mathrm{nF}}=\text { concentration polarization slope (volts) }
\end{gathered}
$$

As the Tafel Equation is an expanded form of Ohm's Law, the anodizing process can be viewed as the operation of an electrical circuit [2], with a portion of the reaction governed by the anode surface and the other by the anodizing electrolyte; the sum is the oxidation potential for the anodizing reaction. Each parameter of the Tafel Equation translates to a process parameter of the anodizing process, which in turn is reflected in the structural characteristics of the anodic aluminum oxide (AAO).

As one considers each parameter of the Tafel Equation in relation to the anodizing process, it is clear that those which are inherently most complex and influence the resultant potential of the circuit most, are the resistance components which comprise the exchange current density, which is affected by both the aluminum substrate and the electrolyte, and includes the temperature. More specifically, the exchange current density is a function of the activation energies that affect changes in circuit resistance as a result of the aluminum alloy chemistry and surface microstructural condition of the substrate; the electrolyte chemistry and concentration; and, the process temperature. 
Whether anodizing a complex die cast alloy for automotive applications or a high purity foil for AAO patterning, the recognition of the relationships between the AAO structure, the microstructure of the aluminum substrate from which it is grown, and the Tafel equation, is consequent in making anodizing an engineering process, one that enables tuning the structure such that it yields distinct characteristics to fulfill design and application requirements.

\section{Modeling Anodic Oxide Growth}

In 1963 Deal and Grove showed that the oxidation reaction kinetics for silicon are controlled by 1) the thermodynamics at the substrate surface and 2) by diffusion [3]. It is no different for the oxidation of aluminum.

Surface finishing reactions and therefore aluminum anodizing can be represented by the Tafel equation [1]. See equation (1). By changing the values for the parameters of the equation, the process parameters for the anodizing reaction are changed, and therefore the finish structure and the finish properties are changed.

$$
\mathrm{N}=\beta_{\mathrm{a}} \frac{\log \mathrm{i}_{\mathrm{a}}}{\mathrm{i}_{\mathrm{o}}}+\frac{2.3 \mathrm{RT}}{\mathrm{nF}} \log \frac{\left(1-\mathrm{i}_{\mathrm{a}}\right.}{\mathrm{i}_{\mathrm{o}}}
$$

A perhaps intuitive simplification of the Tafel equation is that it is an expanded form of Ohm's first Law, with each product representing a portion of the electrochemical reaction:

$$
\mathrm{V}=\mathrm{iR}
$$

The first part of the Tafel equation represents surface activation polarization:

$$
\mathrm{N}_{\text {surface }}=\beta_{\mathrm{a}} \log \frac{\mathrm{i}_{\mathrm{a}}}{\mathrm{i}_{\mathrm{o}}}
$$

The product represents the relationship between activation polarization, $\mathrm{N}_{\text {surface }}$, and the rate of the corrosion reaction by the current density, $\mathrm{i}_{\mathrm{a}}$. Under equilibrium conditions, $\beta_{\mathrm{a}}$ is a constant for a given metal and environment and is temperature dependent, and is typically around $0.1 \mathrm{~V}$. Since an electrical bias is employed to drive anodizing, it is a nonequilibrium process, so the slope $\beta_{\mathrm{a}}$ (the surface polarization) becomes more important. Under nonequilibrium conditions, $\beta_{\mathrm{a}}$ can be regarded as an average slope, or resistance of the aluminum substrate based upon the alloy composition and metallurgical condition, the size of the anode, and the temperature.

Consideration of the current density parameters, $i_{a}$ and $i_{o}$, individually, in terms of oxide growth elucidates their role as a ratio. The applied current density, $\mathrm{i}_{\mathrm{a}}$, drives electron flow and diffusion of metal ions to the substrate-AAO interface, enabling AAO growth. This can be an applied value, if the circuit is galvanostatic, or a resultant value, if the circuit is potentiostatic.

Interfacial resistance effects are reflected in the ever-changing exchange current density, $\mathrm{i}_{\mathrm{o}}$, which fluctuates with changes in interfacial resistance due the changing chemical potential at the interface between the aluminum and the growing oxide. Changes in $i_{0}$ occur as the reaction proceeds and can vary significantly at the interface because of alloying additions, variations in condition of the microstructure (induced through heat treatment, for example) or other microstructural defects, such as excessive cold work, precipitation, or manufacturing process failure at the surface-AAO interface that affect the local activation energy required for the corrosion reaction to continue. The increasing resistance of the growing oxide also changes $\mathrm{i}_{\mathrm{o}}$.

As a ratio, the current density drives both parts of the equation - without it, the finish would not grow beyond a monolayer because the applied bias drives the ions through forming ionic solid which becomes the anodic oxide. An explanation for this is that in all types of aluminum anodizing, regardless of alloy or electrolyte, the thermodynamics of the process seek to oxidize free aluminum; alloying elements and nonmetallic inclusions do not anodize. Electron flow stimulates the ion flow to the interface necessary for oxide growth. Variations in surface charge that deviate from the total charge necessary to anodize due to alloying elements, precipitates, and non-metallic inclusions affect whether nucleation will take place as well as the kinetics of the corrosion that follows. Similarly, surface topographical variations such as sharp edges, machined burrs and texture variations alter the oxidation potential response.

So long as the exchange current density is different from the applied current density, oxidation will go forward. The moment they are equivalent, the anodizing reaction will stop.

In anodizing, the concentration polarization of the electrolyte cannot be ignored as it is typically under equilibrium conditions. The interface created between the substrate surface and the electrolyte is the most important combination of variables that govern the structure and properties of the finished anodic oxide. These are set forth in the second part of the Tafel equation which represents the concentration polarization of the electrolyte.

$$
\mathrm{N}_{\text {solution }}=\frac{2.3 \mathrm{RT} \log }{\mathrm{nF}} \frac{\left(1-\mathrm{i}_{\mathrm{a}}\right)}{\mathrm{i}_{\mathrm{o}}}
$$


In this part of the circuit, the conditions for anodic oxide nucleation are set forth. Together with the surface microstructure, the slope term of the relationship, solution conductivity, which is governed by its composition and concentration; i.e. what acid comprises the electrolyte, and what, if any, additives are included in the electrolyte, dictate the surface activity of the electrolyte on the substrate.

Temperature, together with the surface polarization in the first half of the equation, determines the number and therefore the spacing of each oxide nucleation point that grow and develop into the individual cells which comprise the AAO. A high temperature process will have more nucleation points per unit grain than a low temperature process; a low current density process will have more nucleation points than high current density process.

\section{Chemical Potential and the Forming Anodic Oxide}

Non-aluminum alloy constituents within the substrate such as: alloy additions soluble in the aluminum substrate (phases); intermetallic compounds, which vary in coherency with the substrate; precipitates, which are incoherent with respect to the substrate microstructure; their interfaces and grain boundaries, as well as atomic level defects such as dislocation loops, kinks and ledges, all change the local chemical potential of the substrate [5]. Local changes in chemical potential will result in changes in local interfacial resistance that can be modeled in the surface polarization portion of the Tafel Equation by making the exchange current density, $\mathrm{i}_{\mathrm{o}}$, more complex.

Therefore,

$$
\mathrm{N}_{\text {surface }}=\beta_{\mathrm{a}} \frac{\log \frac{\mathrm{i}_{\mathrm{a}}}{\mathrm{i}_{\mathrm{o}}}}{}
$$

becomes

$$
\mathrm{N}_{\text {local }}=\beta_{\mathrm{a}} \log \mathrm{i}_{\mathrm{a}} \frac{\mathrm{R}_{\text {local }}}{\boldsymbol{\mu}_{\text {local }}}
$$

Where $\mu_{\text {local }}=$ local chemical potential in an area on the substrate, and

$\mathrm{R}_{\text {local }}=$ the local surface resistance due to substrate chemical and/or structural change.

If $i_{o}$ is larger due to increased surface resistance because of changes in local chemical potential, since $\beta_{a}$ is constant, $N_{\text {local }}$ will be smaller, and the reaction kinetics in this area will be slower.

Although the average $\mathrm{N}_{\text {surface }}$ governs the total anodizing reaction, and $\beta_{\mathrm{a}}$ is a characteristic of the metal, variations in $\mu_{\text {local }}$ can induce localized resistance heating which may result in finish defects such as increased oxide surface roughness, variations in surface appearance, and burning.

Because anodizing parameters are suited for the formation of aluminum oxide, increased surface resistance due to local side reactions caused by microstructural defects will change the interfacial resistance and the exchange current density will change; oxide growth rate will be retarded, and depending on the increase of the resistance, finish defects can occur. The level and concentration of alloy additions, constituent segregation, and defects, compound resistance effects therefore impact the quality of the surface finish.

The main effect that local variations in chemical potential, due to substrate microstructural variations, have on the anodizing process is on the exchange current density, which causes local variations in the reaction rate, and therefore increased interfacial resistance heating at various spots over the substrate surface. It is important to realize that compositional variations can occur within the same alloy specification. This is why components manufactured from the same alloy, through the same anodizing process, on the same rack, sometimes at the same time; exhibit different finish appearances, and sometimes different finish performance.

\section{Anodizing as a Corrosion Process}

Because the anodizing reaction is one of substrate (anode) consumption with simultaneous oxide growth, the nature of the total layer formed must be considered as a corrosion process in order to understand how the native oxide can be developed to form the ordered microstructure recognized as AAO.

Aluminum and aluminum alloys corrode to form a passive film in protonic acids which oxidize; for example in sulfuric acid, chromic acid, oxalic acid, phosphoric acid. Aluminum corrodes in these acids by the formation of oxide flakes which cover the surface to make a passive film. Acid chloride solutions tend to break down the passive film on aluminum and initiate localized corrosion by way of pitting [4].

On ideal surfaces, without alloy additions, atomic level defects or other surface contamination, AAO initiates at nuclei whose spacing is ordered and set by the surface polarization. There are several nuclei per grain aluminum, and, the number of nuclei is governed by the temperature of the anodizing process; specifically, lower temperature processes will exhibit fewer nuclei than higher temperature processes. As the corrosion process continues to favor aluminum oxidation, the nuclei grow outward, consuming the substrate with flake-like corrosion products that grow and coarsen (become larger and thicker) to develop a contiguous passive layer comprised of many ordered oxide flakes. The initial reconstructed ideal surface oxide layer resembles a hexagonal bubble layer (Figure 1). 

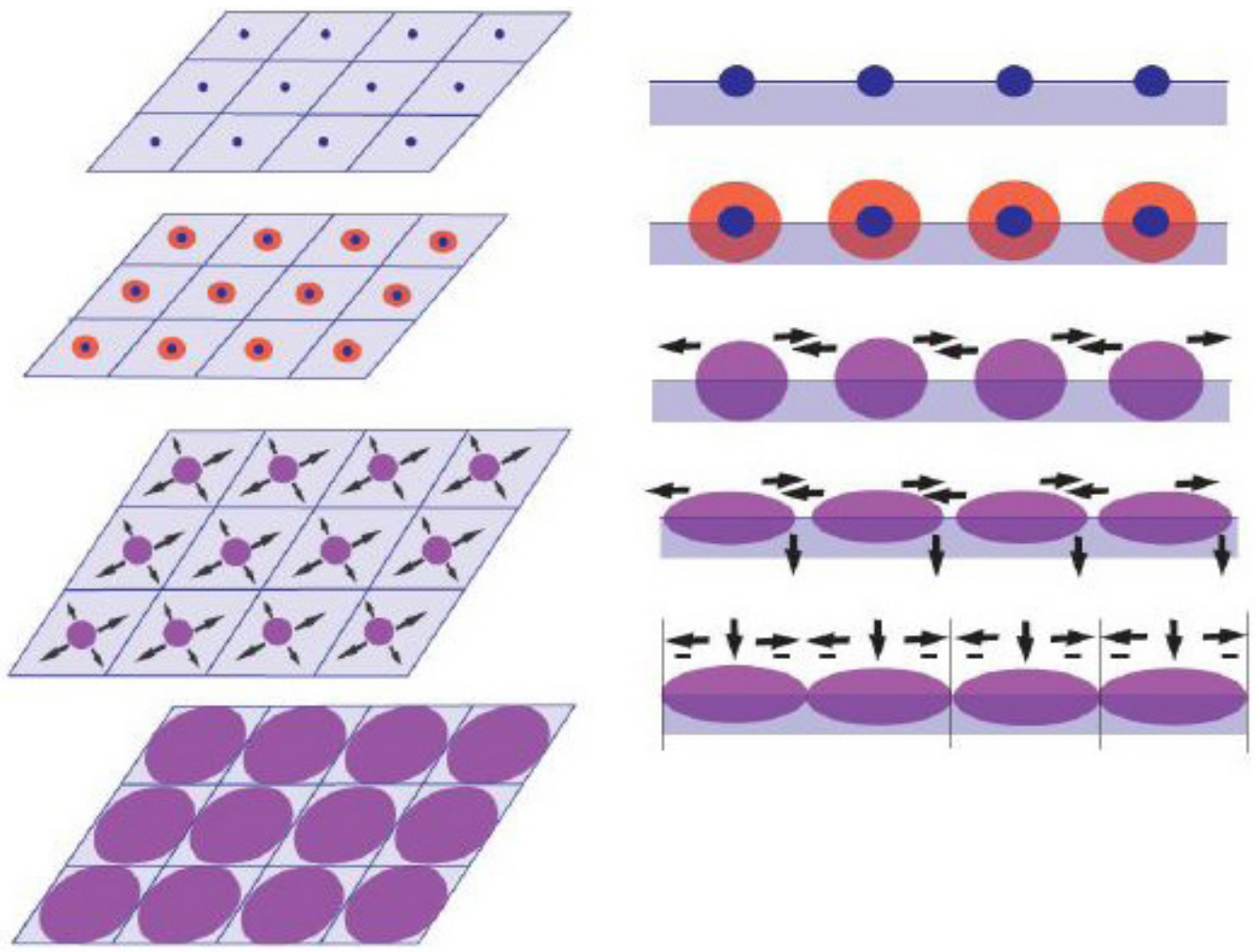

Figure 1: Two-dimensional schematic of ideal surface reconstruction during AAO nucleation and growth. The schematic is both planar and cross sectional, showing that as the nuclei grow and develop, the oxide coarsens, consuming the aluminum substrate both laterally (as shown by the horizontal arrows), and into the bulk of the substrate (as shown by the downward arrows). When the surface is completely reconstructed, the oxide has assembled itself into a layer of hexagonal flakes.

\section{Anodic Oxide Growth}

Oxide growth requires diffusion of oxygen ions from the environment external to the aluminum substrate (the electrolyte) and the metal ions from the aluminum through the forming layer. Without an applied electrical bias, the initial oxide layer would grow in excess of a monolayer to an equilibrium thickness referred to as "native oxide" or a passive layer, and growth would stop [6]. In addition, without an applied electrical bias, the nucleation of the oxide would tend to be entirely preferential; in other words, governed by the surface microstructure. By imposing an external electrical bias to the substrate, surface polarization overrides the resistance of surface phenomena which determine preferential nucleation and produces regular spacing of the oxide nuclei which develop into the highly ordered AAO (Figure 2).
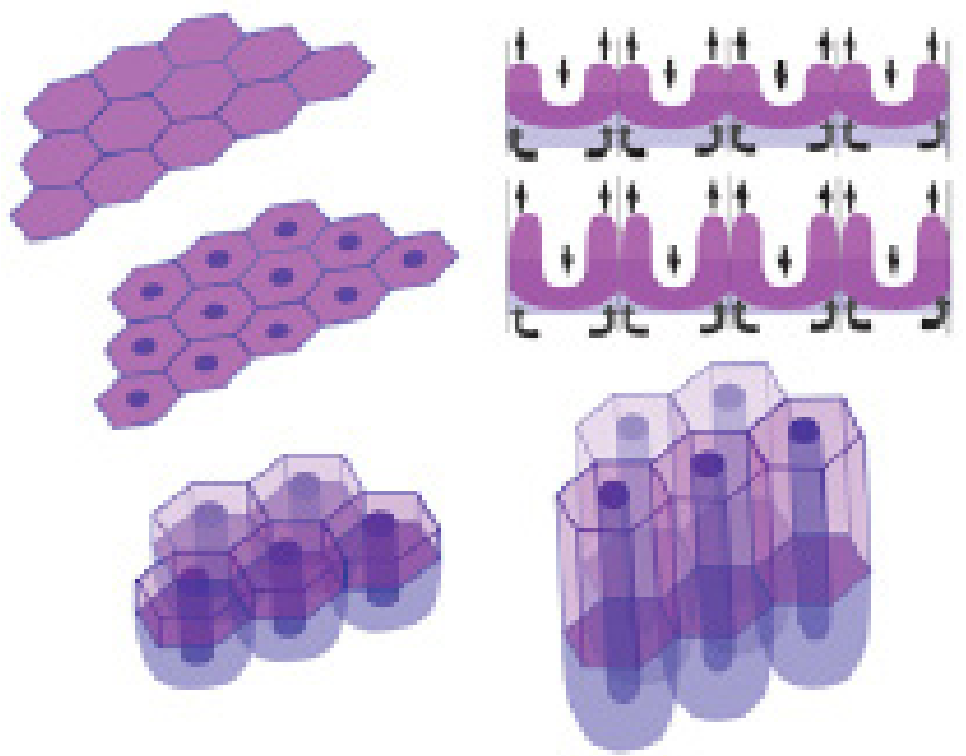

Figure 2: Two-dimensional schematic continued from Figure 1, showing AAO growth following surface reconstruction. The schematic is both planar and cross sectional. Individual corrosion nuclei continue to grow with the imposition of an external electrical bias, impinging on one another, growing upward while consuming the aluminum substrate. The central pore is sustained by the polarization of the individual corrosion "flakes" [7]. 
In nature, preferential nuclei, formed at sites on the substrate surface where the energy required for adsorption is lowest, grow and develop as long as there is available free aluminum to oxidize, and the thermodynamic work function of the metal is such that the oxidation reaction is favored [5]. Work function can be defined as the difference in energy between the electrostatic potential outside the forming oxide layer and the electrochemical potential of the anode. As long as there is a driving force, corrosion proceeds on the substrate surface and lateral oxide growth occurs as the individual nuclei consume the aluminum, reconstructing the surface as an oxide monolayer. Under atmospheric conditions, the oxidation reaction ceases as the monolayer exhibits continuity, causing the work function of the aluminum to reach equilibrium (equal zero), yet it begins again if it is scratched or otherwise disturbed, a characteristic referred to as "self-healing" or passive.

With a deliberate increase in work function, such as through the introduction of a chemical contaminant to the atmosphere or through the use of an electrolyte (as in corrosion or anodizing) and through the imposition of an external electrical bias (as in anodizing) and/or a change in temperature (as in corrosion or anodizing), spacing of the individual nuclei is set and the oxide network grows beyond a passive layer. The oxide nuclei become a network of hydrated, charged flakes that impinge on one another, establishing the hexagonal basis structure that is thermodynamically of lowest energy.

However, as the reaction conditions favor oxidation, the corrosion reaction continues, and after the oxide flakes impinge on one another, outward growth of the oxide begins. The opposing edges of impinging flakes repel one another and grow outward from the substrate surface; forming a unique network of nanoscale corrosion cells. Each cell is comprised of the nucleation point at its rounded base; the flakes grow to become column walls which circumscribe a central pore, which has been created by the repulsive forces of the field effects which sustain the finish growth. Runge first proposed oxide growth by way of the formation of discrete oxide corrosion cells in 2000 [7]. Later, Skeldon et al discussed finish growth via "field assisted alumina flow" [8]. Both theories suggest that pore formation is a function of oxide growth and movement by way of field effects rather than of dissolution by the electrolyte (Figure 3).

(A)

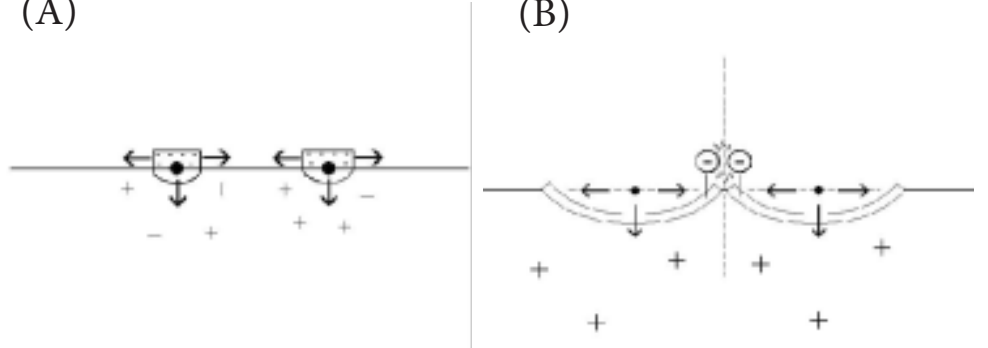

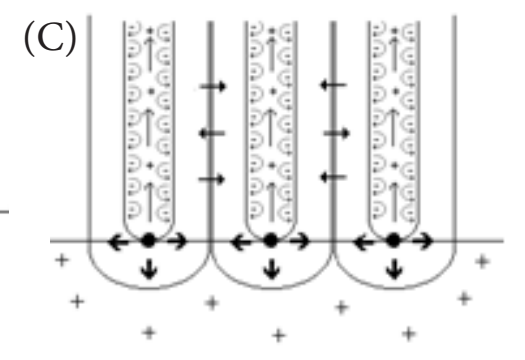

Figure 3: Schematic summarizing AAO nucleation and growth. A: Preferential nuclei form the base of pore. B: Repulsive forces between similarly charged oxide "flakes" foster outward growth and initiate pore formation. C: Mass transport and diffusion across column walls form knitlines while repulsive forces within pore maintain the erect nature of the column and dynamic flow of the electrolyte.

\section{The Importance of the Central Pore}

The feature critical to the forming anodic film is its central pore. As the base network of oxide flakes impinge on one another and grow outward, field effects created on the inside surface of the flakes also repel such that the erect nature of the network is maintained from the inside out. The field effects, which reflect the resultant corrosion potential, dictate the pore diameter.

The inside surface of the cell wall (pore surface) becomes a dynamic surface as the oxidation reaction continues. As the electrolyte reacts through the growing porous structure, the anion, which is in part oxygen, reacts with the aluminum. While the AAO structure grows at the expense of the substrate, the pore surface becomes a surface for ion exchange, with the electrolyte anions migrating into the structure, and non-aluminum metal ions (free alloying elements), dissolved aluminum, and hydrogen ions as $\mathrm{H}+$ as well as hydrogen gas diffusing back through the oxide structure and ejecting from the central pore. Alloy ions that are more cathodic to aluminum, will be cathodically protected during anodizing (aluminum is the sacrificial anode) and remain metallic through the anodizing process. They will either be entrained at the interface or diffuse through the forming AAO and be expelled into the electrolyte.

In addition to exposure time, the changing exchange current density can limit the finish thickness. As the field effects diminish with oxide growth, due to climbing resistance as the finish thickness increases, ion exchange within the columnar structure can no longer be maintained as $i_{0}$ approaches $i_{a}$, and the work function of the anode approaches zero. At this point, the oxidation reaction no longer dominates and adsorption of less reactive species, such as dissolved cations and other ionic contamination, may occur on the pore surface.

\section{The Formation of Knitlines}

To complete the corrosion approach to anodic oxide formation, the outside surface of the cell walls must also be considered. As the oxide flakes impinge and grow outward, because there is no dynamic flux or ion flow across the cell walls, as in the pores, it is apparent that diffusion occurs across the outer wall "knitting" the structure together. The stability and robustness of the final structure hinge on this characteristic of the AAO structure, as it is the only direct avenue from the surface of the oxide to the aluminum substrate. 


\section{The Anodizing Process and AAO Structure Conventions}

The total anodizing voltage, $\mathrm{N}$, must exceed the standard oxidation potential of aluminum (1.6 volts), for the anodizing reaction to proceed. The surface polarization, set by the voltage, governs the regular spacing of the individual oxide cells that comprise the AAO network. If $\mathrm{N}$ is large, the cell nuclei will be spaced further apart, and there will be fewer cells per unit grain of substrate surface than if $\mathrm{N}$ is low. The converse is also true. The significance of the spacing develops as the oxide grows, because the diameter and wall thickness of each discrete AAO cell is determined by the spacing.

Current density, $i$, (amps per unit surface area of substrate) sets the growth rate of the anodizing reaction. If current density is high, the growth rate is fast, and the time to oxide flake impingement is short. Consequently, the AAO cell walls will be thinner when the current density is higher. A lower current density will result in a thicker cell wall, because there is more time before each oxide flake impinges on its nearest neighbors that are growing at the same rate. From the moment the oxide flakes impinge, the cell walls cease to coarsen (cease to become thicker) because substrate consumption by the oxide is translated to upward rather than outward growth. At this point, the characteristic central pore begins to form.

Because a higher current density for a given surface resistance will result in a higher potential response, once the flakes impinge and begin to grow upward, the repulsive forces created by the electric field effects within the central pore will be greater, sustaining a wider pore and a corresponding higher residual stress within the AAO structure. The converse follows as well, with AAO grown at a lower current density (for the same substrate $\left(\beta_{\mathrm{a}}\right)$ and electrolyte as the higher current density example) will have a narrower central pore, and the AAO structure will have a lower residual stress (Figure 4).

Process temperature plays a role in the number of oxide cells that nucleate per unit grain. Simply put, at higher temperature, there are more nuclei per unit grain and the opposite holds true for processes run at lower temperature. As the process continues, the heat of reaction at the interface that includes the substrate resistance, especially with complex alloys, requires the electrolyte be chilled to prevent dissolution of the oxide as it grows.
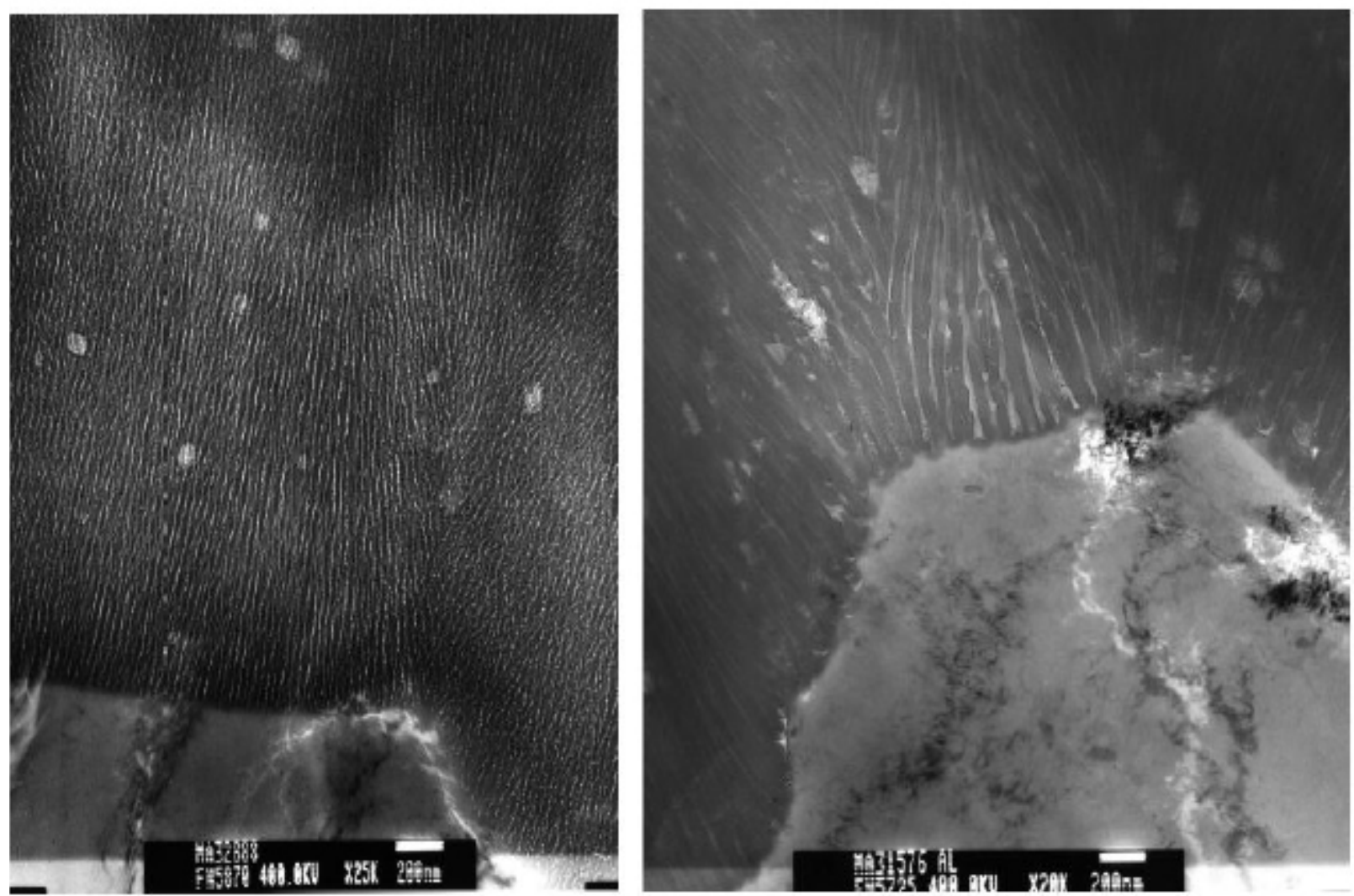

Figure 4: By modifying process parameters of temperature and current density, obvious differences in the AAO structure are clearly developed. The structure on the left was processed at $20^{\circ} \mathrm{C}$, at $1.2 \mathrm{amps} / \mathrm{dm}^{2}$ and with a $120 \mathrm{~g} / \mathrm{l}$ sulfuric acid electrolyte. The structure on the right was processed at $0{ }^{\circ} \mathrm{C}$, at 2.2 amps/dm ${ }^{2}$ and with a $220 \mathrm{~g} / \mathrm{l}$ sulfuric acid electrolyte. The substrate material for both samples was aluminum alloy $6061 \mathrm{~T} 6$ (AlMgSi1). Note the wider pore and thicker cell walls for the structure on the right. 


\section{Conclusion}

As anodizing proceeds, the aluminum substrate is consumed and a reaction product of self-assembling individual corrosion cells with process-dependent structural characteristics come together to form the highly ordered ionic solid known as anodic aluminum oxide. The ordered columnar structure of the anodic oxide is the result of lateral oxide growth from nuclei that develop on the polarized aluminum surface in oxidizing solutions such as sulfuric acid. Lateral growth of the oxide occurs as the electrical bias is applied, and results in impingement of growing oxide "flakes" on one another. The repulsive forces of the similarly charged flakes foster outward growth of the oxide, forming a central pore in each cell that comprises the total anodic oxide. Since the aluminum substrate is consumed to develop the oxide, by definition, anodizing is a controlled corrosion process.

The Tafel Equation links corrosion science and the anodizing process. By relating the anodizing process parameters to the parameters of the Tafel Equation, a connection between the substrate characteristics, the anodizing process and the AAO structural characteristics can be clearly explained.

Each anodizing process yields AAO structures with characteristics reflecting the complexity of the alloy chemistry, manufacturing technique and anodizing electrolyte; however, all resultant AAO structural characteristics follow the trends predicted by the Tafel Equation.

Only the anodic current density, $\mathrm{i}_{\mathrm{a}}$, drives AAO growth; however, of all the parameters discussed, changes in resistance due to chemical potential variations caused by surface and solution resistance, are by far the most important aspects of the anodizing process. These variations directly affect the exchange current density, $\mathrm{i}_{\mathrm{o}}$, which is a function of the activation energies for the oxidation reaction. Therefore, interfacial resistance impacts the structure of the AAO and can produce deviation from the usual trends in structural characteristics.

Whether anodizing a complex die cast alloy for automotive applications or a high purity foil for AAO patterning, recognizing the relationships between the AAO structure, the structure of the aluminum substrate from which it is grown, and the Tafel equation is consequent in making anodizing an engineering process, one that enables tuning the structure such that it yields distinct characteristics to fulfill design and application requirements.

\section{Acknowledgement}

The author would like to acknowledge Auriel Garza of Southern Methodist University in Dallas, Texas for the schematic artwork used in Figures 1 and 2. Dr. Stephen B. Rice, formerly of McCrone Associates, Inc. in Westmont, Illinois, USA is acknowledged for providing the TEM images in figure 4. Much grateful acknowledgement is given to Mr. Larry Chesterfield of Anodizing Technologies, Inc., for his thoughtful criticism of this article.

\section{References}

1. Uhlig HH (1985) Corrosion and Corrosion Control. Wiley-Interscience, New York, USA.

2. Deal BE, Grove AS (1965) General Relationship for the Thermal Oxidation of Silicon. J Appl Phys 36.

3. Bard AJ, Faulkner LR (2001) Electrochemical Methods, Fundamentals and Applications, (2nd Edition), Wiley, New York.

4. Jones Denny A (1995) Principles and Prevention of Corrosion, (2nd Edition), Prentice Hall, Upper Saddle River, NJ.

5. Murr L (1975) Interfacial Phenomena in Metals and Alloys. Addison-Wesley Publishing Company, Boston, USA.

6. Lupis CHP (1983) Chemical Thermodynamics of Materials, Elsevier Science Publishing Company, New York, USA.

7. Runge JM, Pomis AJ (2000) Anodic Oxide Film Formation Relating Mechanism to Composition and Structure. Proceedings ASST, Illinois, USA.

8. Skeldon P, Thompson GE, Garcia-Vergara SJ, Iglesias-Rubianes L, Blanco-Pinzon CE (2006) A Tracer Study of Porous Anodic Alumina. Electrochemical and

Solid State Letters 9: B47- 51.

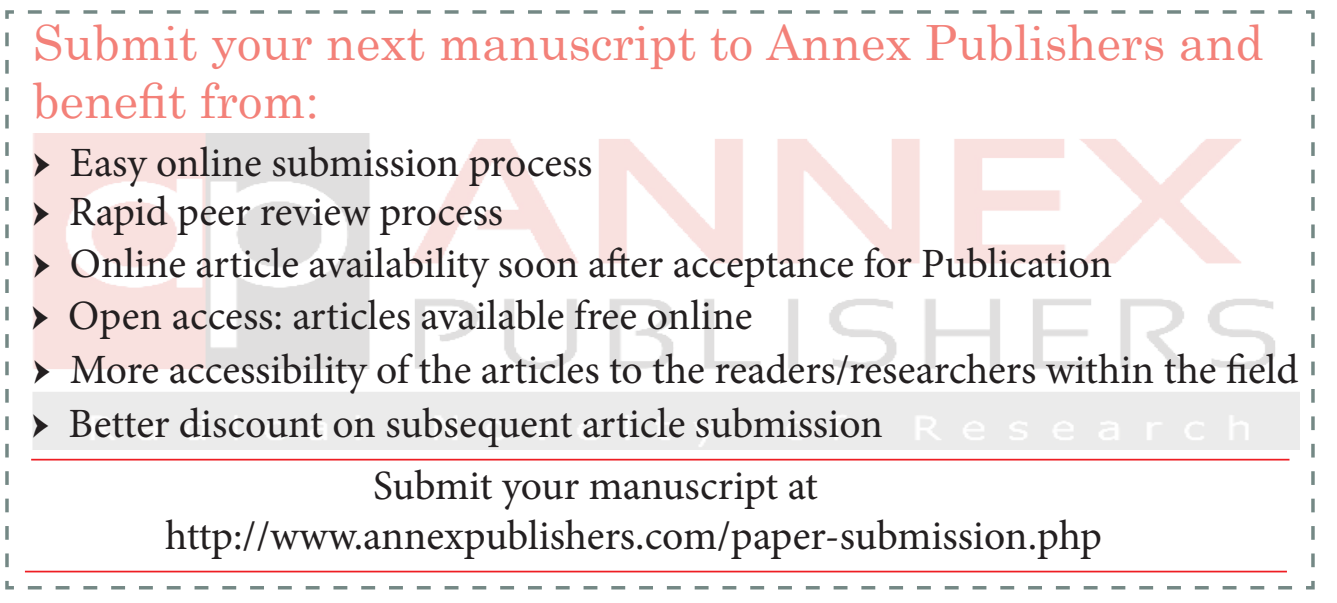

